\title{
Robust impulse-to-peak synthesis: Application to the control of an aerospace launcher
}

\author{
D. Arzelier, D. Peaucelle \\ LAAS-CNRS, 7 Avenue du Colonel Roche, \\ 31077 Toulouse, Cedex 4, France \\ emails: arzelier@laas.fr, peaucell@laas.fr
}

December 15, 2003

\begin{abstract}
A robust synthesis problem via state-feedback involving impulse-to-peak performances is investigated. Even when considering models without uncertainty, the impulse-to-peak performance cannot be computed exactly and a somewhat conservative bound may be computed by LMI optimization [15]. Our goal is first to tighten the bounds obtained in the previous reference using the quadratic stability framework when the state-space matrices are known to belong to a polytope of matrices. In a second stage, the robust state-feedback synthesis problem is tackled in the same setup for LTI discrete-time polytopic systems. These results are then applied to control the attitude of a space launcher during the atmospheric flight.
\end{abstract}

Keywords: Impulse-to-peak performance, robust state-feedback synthesis, LMI optimization, Aerospace launcher.

\section{Introduction and problem formulation}

It is well-known that $\mathcal{H}_{\infty}$ synthesis guarantees robust stability in the face of worst-case disturbances while $\mathcal{H}_{2}$ synthesis is more adapted to deal with nominal performance. When imposing transient specifications (overshoot, settling time), a less known approach is to guarantee a bound on the peak impulse response. Even when considering models without uncertainty, the impulse-to-peak performance cannot be computed exactly and a somewhat conservative bound may be computed by LMI optimization [2] and [15] for the multiobjective context. In the presence of plant perturbations the analysis as well as the synthesis problem are in general open problems, see [17] and [18] for LFT uncertain structure.

Our goal is first to tighten the computable bound on the worst case impulse-to-peak performance when the system matrices are known to belong to a polytope defined by its vertices. It is well-known that using a vertexization argument and the framework of quadratic stability, rough bounds may be easily obtained via LMI optimization [2]. New conditions based on the introduction of additional slack variables and already used in different contexts [7], [11], [14], [13]are proposed to compute tighter bounds than the ones developed in the quadratic setup. These conditions are then relaxed to tackle the problem of robust impulse-to-peak state-feedback synthesis for polytopic uncertain systems.

A final important part of the paper is dedicated to the application of these results to the problem of the robust control of a space launcher. During its atmospheric flight, the non-stationary rigid dynamics of a space launcher have to be regulated in terms of angle of attack [9], [10], [4], [20]. In particular, this paper focuses on the mandatory requirement on the worst case peak amplitude of the angle of attack when a typical wind profile is applied. The aerodynamic coefficient and the thruster efficiency are uncertain parameters. Robust impulse-to-peak state-feedback synthesis is therefore applied on this problem and simulation results are given.

Let the LTI discrete plant $\Sigma$ be given by its state-space minimal realization:

$$
\left[\begin{array}{c}
x_{k+1} \\
z_{k}
\end{array}\right]=\left[\begin{array}{ccc}
A & B_{1} & B \\
C_{1} & D_{11} & D
\end{array}\right]\left[\begin{array}{c}
x_{k} \\
w_{k} \\
u_{k}
\end{array}\right]
$$

where $x \in \mathbb{R}^{n}$ is the state vector, $w \in \mathbb{R}^{m_{w}}$ is the disturbance vector, $u \in \mathbb{R}^{m_{u}}$ is the input vector and $z \in \mathbb{R}^{r_{z}}$ is the controlled output vector. The model is supposed to be uncertain since the system matrices 
are assumed to belong to a polytope of matrices defined as the convex hull of $N$ vertices.

$$
\left[\begin{array}{ccc}
A & B_{1} & B \\
C_{1} & D_{11} & D
\end{array}\right] \in \Omega=\operatorname{co}\left\{\left[\begin{array}{ccc}
A^{[1]} & B_{1}^{[1]} & B^{[1]} \\
C_{1}^{[1]} & D_{11}^{[1]} & D^{[1]}
\end{array}\right], \cdots,\left[\begin{array}{ccc}
A^{[N]} & B_{1}^{[N]} & B^{[N]} \\
C_{1}^{[N]} & D_{11}^{[N]} & D^{[N]}
\end{array}\right]\right\}
$$

It is assumed that the whole state is measurable such that a state-feedback may be utilized for stabilization and performance requirements. The controller $K$ is given by its state-space realization:

$$
u_{k}=K x_{k}
$$

The closed-loop system $\Sigma \star K$ is given by its state-space equations:

$$
\Sigma \star K \quad \begin{array}{ll}
x_{k+1}=A_{c l} x_{k}+B_{1} w_{k} \quad x(0)=0 \\
z_{k}=C_{1 c l} x_{k}+D_{1} w_{k}
\end{array}
$$

where:

$$
A_{c l}=A+B K \quad C_{1 c l}=C_{1}+D_{1 u} K
$$

This paper focuses on two related problems.

\section{PROBLEM 1 (robust impulse-to-peak control problem)}

Find a robustly stabilizing state-feedback $K \in \mathcal{K}$ such that for all realizations of the model in the polytope $\Omega$ :

$$
\begin{array}{ll}
\min _{\mathbf{K} \in \mathcal{K}} & \gamma_{\boldsymbol{i 2} \boldsymbol{p}} \\
\text { under } & \|\Sigma \star \mathbf{K}\|_{i 2 p}^{2} \leq \gamma_{\boldsymbol{i} \mathbf{p}}
\end{array}
$$

Once a satisfying state-feedback gain $K$ is found, it is interesting to compute the tighter estimate of the worst case impulse-to-peak performance of the closed-loop system.

PROBLEM 2 (robust impulse-to-peak analysis problem)

Find the worst case impulse-to-peak performance of the closed-loop uncertain polytopic model $\Sigma \star K$ for all $\Sigma \in \Omega$.

Surprisingly, except in some particular cases, both these problems are hard to solve analytically. Even if some sub-optimal numerical approach has been developed [15], the reduction of the conservatism of the proposed solution is still a challenging problem in both cases.

\section{LMI formulations of the robust impulse-to-peak analysis prob- lem}

\subsection{Quadratic and extended LMI-based impulse-to-peak test}

As in continuous-time case [2], [15], a conservative LMI characterization may be used to compute a bound on the peak of the impulse response of a polytopic discrete LTI system. For a given state-feedback $K$, the closed-loop discrete-time plant belongs to a polytope of matrices $\Omega_{c l}$.

$$
\left[\begin{array}{ccc}
A_{c l} & B_{1} & B \\
C_{1 c l} & D_{11} & D
\end{array}\right] \in \Omega_{c l}=c o\left\{\left[\begin{array}{ccc}
A_{c l}^{[1]} & B_{1}^{[1]} & B^{[1]} \\
C_{1 c l}^{[1]} & D_{11}^{[1]} & D^{[1]}
\end{array}\right], \cdots,\left[\begin{array}{ccc}
A_{c l}^{[N]} & B_{1}^{[N]} & B^{[N]} \\
C_{1 c l}^{[N]} & D_{11}^{[N]} & D^{[N]}
\end{array}\right]\right\}
$$

Therefore, a sub-optimal solution for problem 2 may be found by solving the LMI optimization problem

\section{THEOREM 1}

If there exists a matrix $\mathbf{P}_{\mathbf{c l}} \in \mathbb{S}^{*+}$ and a scalar $\gamma^{*}$ satisfying for all $i=1, \cdots, N$ :

$$
\begin{array}{ll}
\gamma_{q}^{*}=\min _{\mathbf{P}_{\mathbf{c l}}, \boldsymbol{\gamma}} & \gamma \\
\text { under } & \\
& A_{c l}^{[i]} \mathbf{P}_{\mathbf{c l}} A_{c l}^{[i]^{\prime}}-\mathbf{P}_{\mathbf{c l}}<\mathbf{0} \\
& B_{1}^{[i]} B_{1}^{[i]^{\prime}}-\mathbf{P}_{\mathbf{c l}}<\mathbf{0} \\
& C_{1 c l}^{[i]} \mathbf{P}_{\mathbf{c l}} C_{1 c l}^{[i]^{\prime}}-\gamma \mathbf{1}<\mathbf{0} \\
& D_{1}^{[i]} D_{1}^{[i]^{\prime}}-\gamma \mathbf{1}<\mathbf{0}
\end{array}
$$


then $\left\|z_{k}\right\| \leq \sqrt{\gamma_{q}^{*}} \forall k \geq 0$.

Proof 1

Note that by Schur complement, all the inequalities defining the realizable set of (8) may be equivalently written with respect to $X_{c l}=P_{c l}^{-1}$ and for all realizations of $\Sigma \star K$ in the polytope $\Omega_{c l}$. Subsequent technical developments are rather standard and may be found in the reference [1].

For a moderate number of vertices, this approach rooted in the quadratic stability framework is known to be numerically efficient while giving a rather conservative bound $\gamma_{q}^{*}$. The first contribution of this paper is to propose a tighter bound on the robust impulse-to-peak performance. This bound is computed via the solution of an LMI optimization problem involving extra matrix variables (slack variables) allowing the use of polytopic parameter-dependent Lyapunov functions. Indeed, it is well-known that the main origin of conservatism of the quadratic approach is the use of a single Lyapunov function for the whole domain of uncertainty.

\section{TheOREM 2}

If there exist $N$ positive definite matrices $\mathbf{P}_{\mathbf{c l}}^{[\mathbf{i}]} \in \mathcal{S}_{n}$ and two matrices $\mathbf{F} \in \mathbb{R}^{n \times n}$ and $\mathbf{G} \in \mathbb{R}^{n \times n}$ and a positive scalar $\gamma$ solution to:

$$
\begin{aligned}
\gamma_{F G}^{*}=\min _{\mathbf{P}_{\mathbf{c l}}^{[\mathrm{i}]}, \boldsymbol{\gamma}, \mathbf{G}, \mathbf{F}} & \gamma \\
\text { under } & {\left[\begin{array}{cc}
-\mathbf{P}_{\mathbf{c l}}^{[\mathbf{i}]} & A_{c l}^{[i]} \mathbf{G} \\
\mathbf{G}^{\prime} A_{c l}^{[i]^{\prime}} & \mathbf{P}_{\mathbf{c l}}^{[\mathbf{i}]}-\mathbf{G}-\mathbf{G}^{\prime}
\end{array}\right]<\mathbf{0} } \\
& {\left[\begin{array}{cc}
-\mathbf{P}_{\mathbf{c l}}^{[\mathbf{i}]} & B_{1}^{[i]} \\
B_{1}^{[i]^{\prime}} & -\mathbf{1}
\end{array}\right]<\mathbf{0} } \\
& {\left[\begin{array}{cc}
-\gamma \mathbf{1} & \\
\mathbf{F}^{\prime} C_{1 c l}^{[i]^{\prime}} & \mathbf{P}_{\mathbf{c l}}-\mathbf{F}-\mathbf{F}^{\prime}
\end{array}\right]<\mathbf{0} } \\
& {\left[\begin{array}{cc}
-\gamma \mathbf{1} & D_{1}^{[i]} \\
D_{1}^{[i]^{\prime}} & -\mathbf{1}
\end{array}\right]<\mathbf{0} }
\end{aligned}
$$

then

$$
\sqrt{\gamma_{q}^{*}} \geq \sqrt{\gamma_{F G}^{*}} \geq\left\|z_{k}\right\| \quad \forall k \geq 0
$$

\section{Proof 2}

The proof comes from the application of elimination lemma [16] on the first and third inequality of (8). The inequality $\gamma_{q}^{*} \geq \gamma_{F G}^{*}$ may be proved by noticing that the quadratic test may be retrieved by choosing $P_{c l}^{[i]}=P_{c l}$ for $i=1, \cdots, N, F=P_{c l}$ and $G=P_{c l}$.

\subsection{Numerical example}

The next example is borrowed from [6] (second example). It consists in the discretized longitudinal short period mode dynamics of the F4E fighter aircraft. Four different operating points are considered leading to a polytope with four vertices. The system matrices may be retrieved from the reference above. First, the quadratically stabilizing state-feedback given in [6] is used to robustly stabilize the polytope:

$$
K=\left[\begin{array}{lll}
0.0379 & 0.5714 & -0.3499
\end{array}\right]
$$

We get the following bounds on the closed-loop impulse-to-peak performance:

$$
\sqrt{\gamma_{q}^{*}}=13.4003 \sqrt{\gamma_{F G}^{*}}=11.0054
$$

The improvement is about $18 \%$ for the extended test compared to the quadratic one. This is obtained without much more numerical involvements. 


\section{LMI formulation of the robust impulse-to-peak control problem}

\subsection{Extended impulse-to-peak synthesis condition}

To address the impulse-to-peak performance synthesis problem, we need to relax the previous optimization problem by setting $G=F$. This relaxation is known as the generalized shaping paradigm [13].

THEOREM 3

If there exist $N$ positive definite matrices $\mathbf{P}^{[\mathbf{i}]} \in \mathcal{S}_{n}$, matrices $\mathbf{G} \in \mathbb{R}^{n \times n}, \mathbf{S} \in \mathbb{R}^{m \times n}$, and a scalar $\gamma_{G}^{*} \in \mathbb{R}^{+*}$ such that:

$$
\begin{aligned}
\gamma_{G}^{*}=\min _{\mathbf{G}, \mathbf{P}^{[\mathbf{i}]}, \gamma_{G}} & \gamma \\
& {\left[\begin{array}{cc}
-\mathbf{P}^{[\mathbf{i}]} & A^{[i]} \mathbf{G}+B_{1}^{[i]} \mathbf{S} \\
\star & \mathbf{P}^{[\mathbf{i}]}-\mathbf{G}-\mathbf{G}^{\prime}
\end{array}\right]<\mathbf{0} } \\
& {\left[\begin{array}{cc}
-\mathbf{P}^{[\mathbf{i}]} & B_{1}^{[i]} \\
\star & -\mathbf{1}
\end{array}\right]<\mathbf{0} } \\
& {\left[\begin{array}{cc}
-\gamma \mathbf{1} & C_{1}^{[i]} \mathbf{P}^{[\mathbf{i}]}+D_{1 u}^{[i]} \mathbf{S} \\
\star & \mathbf{P}^{[\mathbf{i}]}-\mathbf{G}-\mathbf{G}^{\prime}
\end{array}\right]<\mathbf{0} } \\
& {\left[\begin{array}{cc}
-\gamma \mathbf{1} & D_{1}^{[i]} \\
\star & -\mathbf{1}
\end{array}\right]<\mathbf{0} }
\end{aligned}
$$

then $K_{G}=S G^{-1}$ is a stabilizing state-feedback and the closed-loop peak value of impulse response of the system is bounded by:

$$
\|z\|_{\infty}<\sqrt{\gamma_{G}^{*}}
$$

ProOF 3

From (9), setting $F=G, P=P_{c l}$ and performing the invertible linearizing change of variables $S=K P$, it is easy to get the LMI optimization problem (12).

\section{$3.2 \quad$ Numerical example}

The same example as the previous section is used here to test the synthesis condition (12).

$$
K_{G}=\left[\begin{array}{lll}
0.0640 & 0.5062 & -0.2546
\end{array}\right] \quad \sqrt{\gamma_{G}^{*}}=10.2938
$$

Analyzing the closed-loop system obtained with $K_{G}$, it is interesting to note that it is not quadratically stable but robustly stable. Performing the extended analysis test on this closed-loop uncertain system leads to compute:

$$
\sqrt{\gamma_{G F}^{*}}=10.2536
$$

This result clearly shows that the relaxation introduced in the synthesis procedure by imposing $F=G$ is not too conservative in this case.

\section{Application to the multiobjective control of an aerospace launcher}

\subsection{Model of the launcher and specifications}

The description of the application is mainly borrowed from [3]. The yaw axis of the space launcher with the associated variables is presented in figure 1. $V$ and $V_{r}$ are the absolute and relative velocity and $W$ is the wind velocity. $i$ is the angle of attack and $\psi$ is the deviation of the launcher from the yaw axis with respect to the guidance attitude reference. One has to design an automatic controller keeping the launcher around its center of gravity which follows the guidance reference trajectory where the control variable is the thruster angle of deflection $\beta$. A simplified linearized model of the rigid part of the launcher (without bending modes 
and actuator dynamics) where the different variables are defined as the deviation angle with respect to the guidance attitude reference $\psi$, the angular velocity $\dot{\psi}$ and the lateral drift rate $\dot{z}$.

$$
\begin{aligned}
& \ddot{\psi}(t)=A_{6}\left(\psi(t)+\frac{\dot{z}(t)-W(t)}{V}\right)+K_{1} \beta(t) \\
& \ddot{z}(t)=a_{1} \psi(t)+a_{2}(\dot{z}(t)-W(t))+a_{3} \beta(t)
\end{aligned}
$$

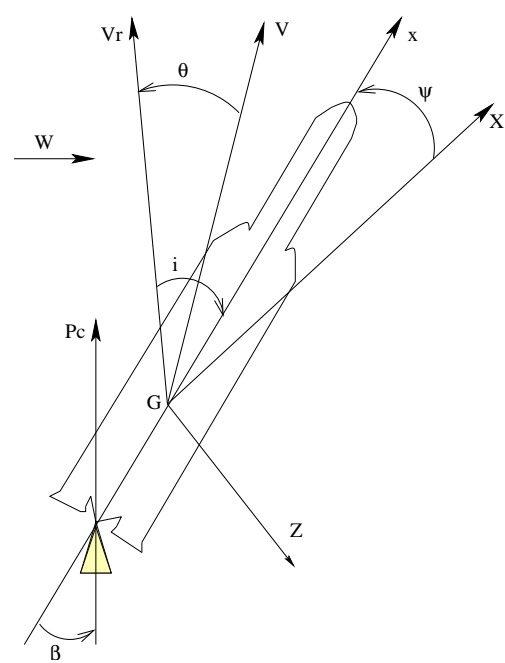

Figure 1: Model of the launcher

Under the assumptions of small angles, the angle of attack equation reads:

$$
i(t)=\psi(t)+\frac{\dot{z}(t)-W(t)}{V}
$$

This model depends on known aerodynamic coefficients $a_{i}, i=1,2,3$ and time-varying coefficients $A_{6}$ the aerodynamic efficiency and $K_{1}$ the thruster efficiency along the flight envelope. Defining the state vector as $x=\left[\begin{array}{lll}\Psi & \dot{\Psi} & \dot{z}\end{array}\right]^{\prime}$, the perturbation input as $W$ and the controlled output as $i$ and assuming that $A_{6}$ and $K_{1}$ are known to be in some interval, $\underline{A}_{6} \leq A_{6} \leq \bar{A}_{6}$ and $\underline{K}_{1} \leq K_{1} \leq \bar{K}_{1}$ we get the following uncertain polytopic state-space model.

$$
\begin{aligned}
& \dot{x}(t)=\left[\begin{array}{ccc}
0 & 1 & 0 \\
A_{6} & 0 & \frac{A_{6}}{V} \\
a_{1} & 0 & a_{2}
\end{array}\right] x(t)+\left[\begin{array}{c}
0 \\
-\frac{A_{6}}{V} \\
-a_{2}
\end{array}\right] W+\left[\begin{array}{c}
0 \\
K_{1} \\
a_{3}
\end{array}\right] u(t) \\
& z(t)=i(t)=\left[\begin{array}{lll}
1 & 0 & \frac{1}{V}
\end{array}\right] x(t)-\frac{1}{V} W
\end{aligned}
$$

To apply the approach proposed in this paper, an uncertain polytopic discrete-time model is needed. Using Euler's first-order approximation for the derivative and a sampling time $T_{s}$, we get the following uncertain polytopic discrete-time model:

$$
\begin{aligned}
& x_{k+1}=A x_{k}+B_{1} w_{k}+B u_{k} \\
& z_{k}=C_{1} x_{k}+D_{11} w_{k}
\end{aligned}
$$

where

$$
\begin{array}{rlr}
A=\left[\begin{array}{ccc}
1 & T_{s} & 0 \\
A_{6} T_{s} & 1 & \frac{A_{6} T_{s}}{V} \\
a_{1} T_{s} & 0 & a_{2} T_{s}+1
\end{array}\right] \quad B_{1}=\left[\begin{array}{c}
0 \\
-\frac{A_{6} T_{s}}{V} \\
-a_{2} T_{s}
\end{array}\right] \quad B=\left[\begin{array}{c}
0 \\
K_{1} T_{s} \\
a_{3} T_{s}
\end{array}\right] \\
C_{1}=\left[\begin{array}{lll}
1 & 0 & \frac{1}{V}
\end{array}\right] & D_{11}=-\frac{1}{V}
\end{array}
$$


Due to the variation of the coefficients $A_{6}$ and $K_{1}$, we get a polytope of matrices with four vertices.

$$
\left[\begin{array}{lll}
A & B_{1} & B
\end{array}\right] \in \Omega=\left\{\left[\begin{array}{lll}
A(\lambda) & B_{1}(\lambda) & B(\lambda)
\end{array}\right]=\sum_{i=1}^{4} \lambda_{i}\left[\begin{array}{ccc}
A^{[i]} & B_{1}^{[i]} & B^{[i]}
\end{array}\right] \quad \sum_{i=1}^{4} \lambda_{i}=1 \quad \lambda_{i} \geq 0\right\}
$$

A robust state-feedback $K_{r}$ minimizing the worst case impulse-to-peak performance is therefore computed by solving LMI optimization problem (12). This robust state-feedback leads to the closed-loop poles plotted in figure 2. A discretization on the range of variation of the coefficients $A_{6}$ and $K_{1}$ has been done to check the robust stability of the closed-loop.

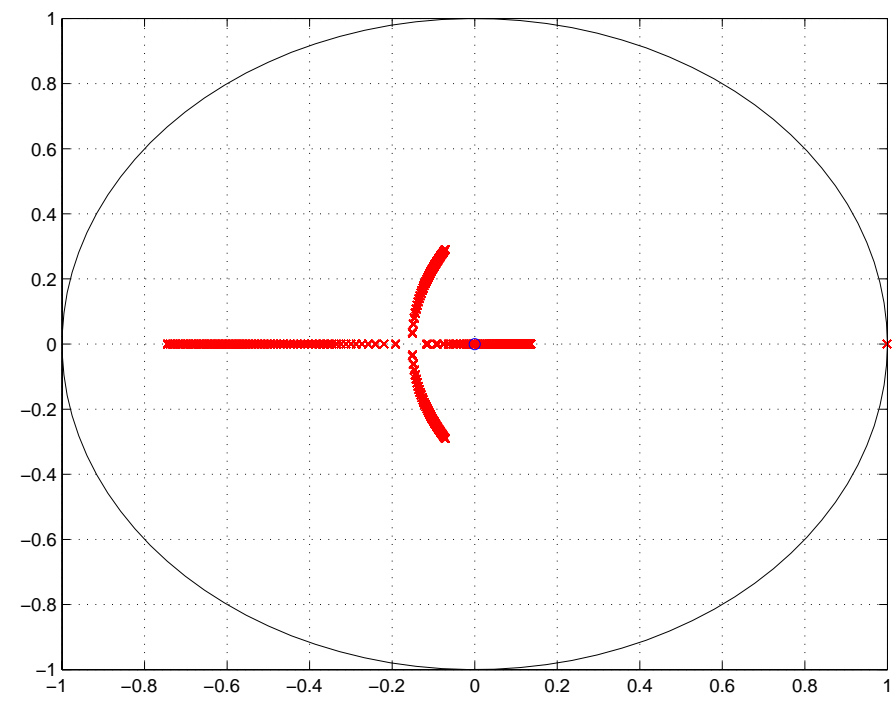

Figure 2: Closed-loop poles

Furthermore, the dynamical performance of the controlled launcher have been simulated in response to the typical wind profile presented in figure 3.

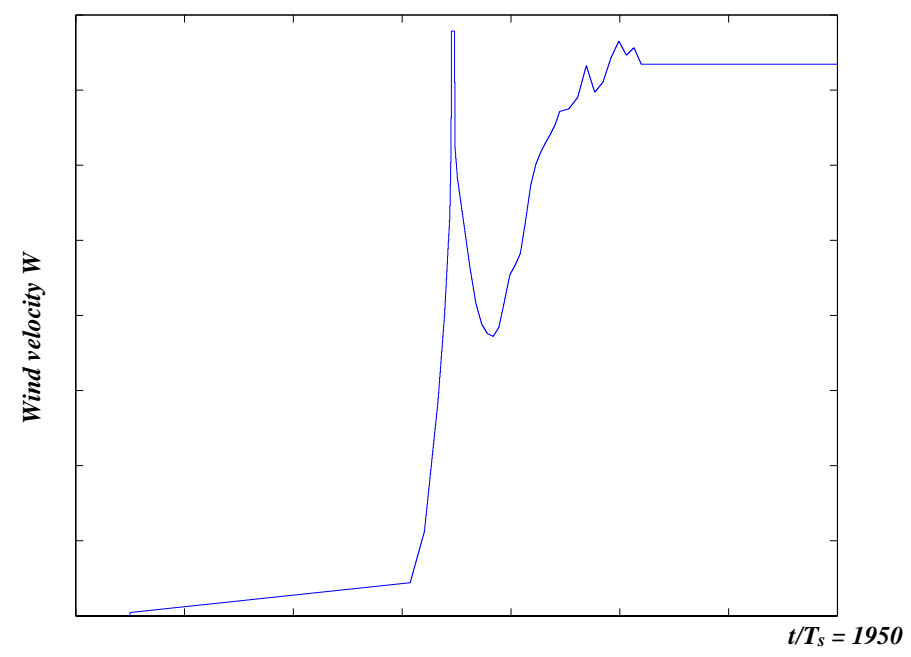

Figure 3: Wind profile

The main time-domain performance specification is defined by maximal and minimal bounds on the peak value of the angle of attack when the wind profile is applied to the launcher. Note also that additional noise signals have been added to the state variables $\psi, \dot{\psi}$ and $\dot{z}$ since they are considered to be measured. 


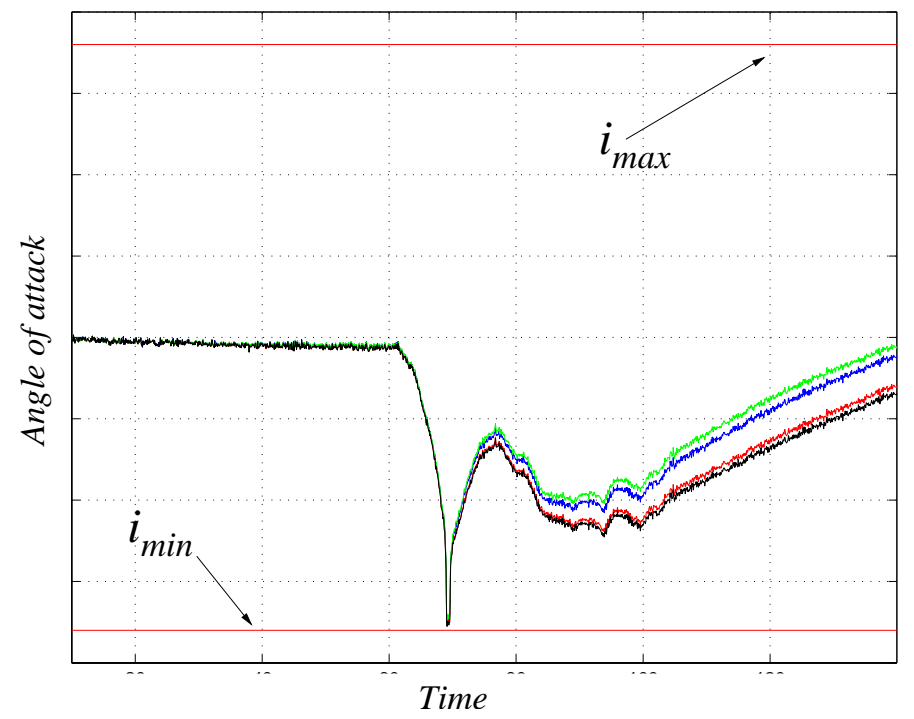

Figure 4: Angle of attack

Four extreme realizations (the vertices of the polytope) of the rigid launcher have been simulated. Figure 4 shows the behavior of the robust impulse-to-peak controller. Even if the angle of attack lies in the required domain of performance, we have no degree of freedom with respect to the bounds. A way to improve the previous design is to introduce a weight $\rho$ in the channel defining the perturbation (wind velocity $W$ ).

$$
\begin{aligned}
& x_{k+1}=A x_{k}+\rho B_{1} w_{k}+B u_{k} \\
& z_{k}=C_{1} x_{k}+\rho D_{11} w_{k}
\end{aligned}
$$

For $\rho=1000$, a slight improvement of the dynamical performance with respect to the peak value of the angle of attack may be obtained (figure 5 .

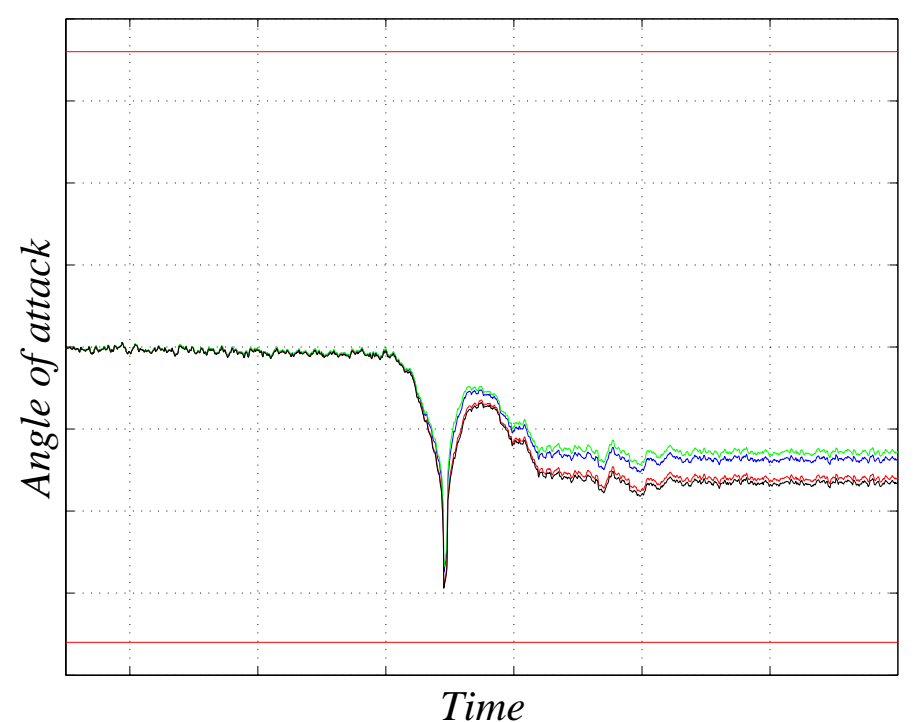

Figure 5: Angle of attack $(\rho=1000)$

The poles-zeros map of the uncertain closed-loop system when coefficients $A_{6}$ and $K_{1}$ are discretized over their range of variations is given in figure 6 


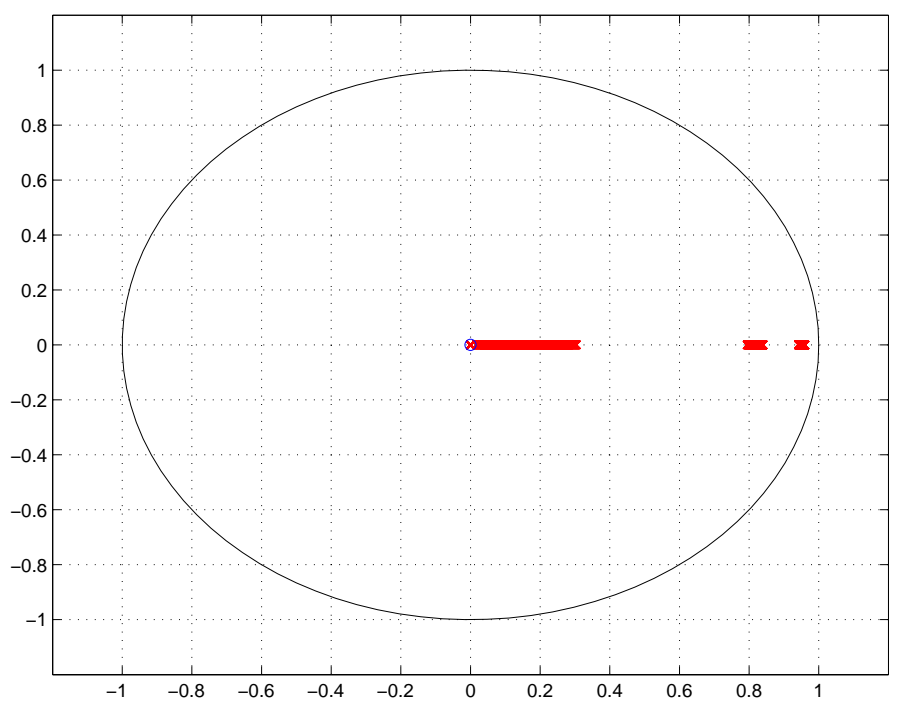

Figure 6: Closed-loop poles $(\rho=1000)$ below.

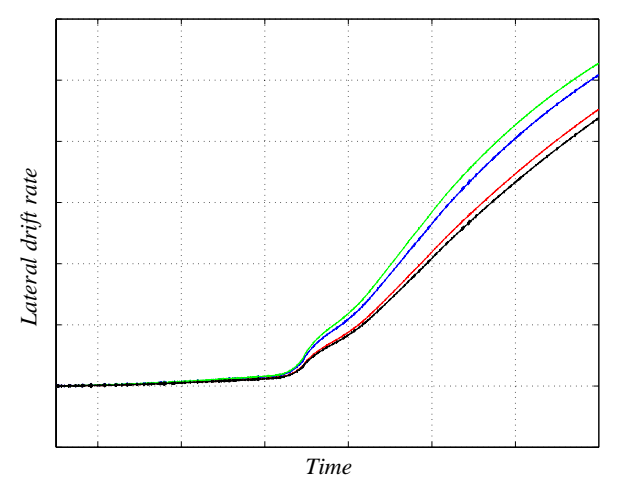

Lateral drift rate $(\rho=1)$

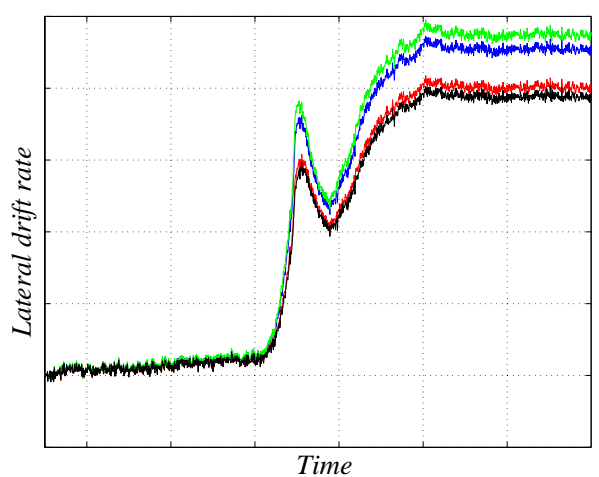

Lateral drift rate $(\rho=1000)$

\section{Conclusions}

New LMI conditions for the analysis of worst case impulse-to-peak performance for polytopic uncertainties as well as for robust state-feedback synthesis have been proposed. It has been shown that this conditions always encompass the existing ones based on the quadratic stability framework. Finally, this approach has been applied to the realistic example of the control of an aerospace launcher. Stress has been laid on the main performance requirement which is expressed in terms of maximal peak value of the angle of attack in response to a specific wind profile. Of course, many other specifications (attenuation of bending modes, consumption criterion, stability margins on the rigid model as well as on the bending modes...) must be considered. In addition, the whole state is not available in practice for control purpose. Only $\psi$ and $\dot{\psi}$ may be measured and the dynamics of the sensors and the actuator have to be taken into account (see the references [9], [3], [4], [20], [1] for details).

In [1], a multiobjective $H_{2} / H_{\infty} /$ impulse-to-peak controller which verifies all the specifications imposed on the launcher during the atmospheric flight is computed via LMI optimization and extended conditions. The parametric uncertainty is not directly tackled as it is in that paper. It is shown here that extended stabilization conditions may be adequately applied to explicitly deal with parametric uncertainty entering the system matrices of the launcher. To extend this type of synthesis to the real practical problem of controlling the launcher, the procedure of synthesis proposed in [20] may be applied with the robust state-feedback computed by (12).

One theoretical and practical perspective to this work would be to generalize the following conditions to the problem of robust dynamical output feedback synthesis for which there does not exist any LMI solution to date. 


\section{References}

[1] D. Arzelier, D. Peaucelle, "Multiobjective $H_{2} / H_{\infty}$ impulse-to-peak synthesis: Application to the control of an aerospace launcher", Proceedings of the 16th IFAC Symposium on Automatic Control in Aerospace, St. Petersburg, June 2004.

[2] S.P. Boyd, L.E. El Ghaoui, E. Feron, V. Balakrishnan, Linear matrix inequalities in system and control theory, SIAM Studies, 1994.

[3] B. Clement, G. Duc, "A multiobjective control algorithm: Application to a launcher with bending modes", Proceedings of the 8th IEEE Mediterranean Conference on Control and Automation, Rio Patras, Greece, 2000.

[4] B. Clement, G. Duc, S. Mauffrey, A. Biard, "Aerospace launch vehicle control: a gain scheduing approach", 15th triennial World Congress, Barcelona, 2002.

[5] P. Gahinet, A. Nemirovski, A. Laub, M. Chilali, LMI Control Toolbox, LMI Control Toolbox User's Guide, 1995.

[6] J.C. Geormel, P.L.D. Peres, S.R. Souza, " $\mathrm{H}_{2}$ guaranteed cost control for uncertain discrete-time linear systems", Int. J. Control Vol. 57, No. 4, pp. 853-864, 1993.

[7] J.C. Geromel, M.C. de Oliveira, L. Hsu, "LMI Characterization of structural and robust stability", Linear Algebra and its Applications, vol. 285, 1-3, pp. 69-80, December 1998.

[8] N. Imbert, B. Clement, "Launcher attitude control: some answers to the robustness issue", 16th IFAC Symposium on Automatic Control in Aerospace, St Petersbourg, Russia, 2004.

[9] S. Mauffrey, M. Schoeller, "Non-stationnary $H_{\infty}$ control for launcher with bending modes", 14th IFAC Symposium on Automatic Control in Aerospace, Seoul, Korea, 1998.

[10] S. Mauffrey, P. Meunier, G. Pigni, A. Biard, I. Rongier, " $H_{\infty}$ control for the ARIANE 5 plus Launcher", 52nd International Astraunotic Congress, Toulouse, France, 2001.

[11] M.C. de Oliveira, J. Bernussou, J.C. Geromel, "A new discrete-time robust stability condition", Systems \& Control Letters, Vol. 37, No. 4, July 1999.

[12] M.C. de Oliveira, J.C. Geromel, L. Hsu, "LMI Characterization of structural and robust stability: the discrete-time case", Linear Algebra and its Applications, Vol. 296, 1-3, pp.27-38, July 1999.

[13] M.C. Oliveira, J. Bernussou, J.C. Geromel, "Extended $H_{2}$ and $H_{\infty}$ norm characterizations and controller parametrizations for discrete-time systems", International Journal of Control, Vol. 75, pp. 666-679, 2002.

[14] D. Peaucelle, D. Arzelier, O. Bachelier, J. Bernussou, "A new robust $\mathcal{D}$-stability condition for real convex polytopic uncertainty", Systems \& Control Letters, vol. 40, 1, may 2000.

[15] C.W. Scherer, P. Gahinet, M. Chilali, "Multiobjective output-feedback control via LMI optimization", IEEE Transactions on Automatic Control, Vol. 42, No. 7, pp. 896-911, 1997.

[16] R.E. Skelton, T. Iwasaki, K. Grigoriadis, A unified algebraic approach to linear control design, Taylor and Francis, 1998.

[17] H. Tokunaga, T. Iwasaki, S. Hara, "Multi-objective robust control with transient specifications", Proceedings of the 35th Conference on Decision and Control, Kobe, Japan, 1996.

[18] H. Tokunaga, T. Iwasaki, S. Hara, "Analysis and synthesis of the robust impulse-to-peak performance", Automatica, Vol. 34, No. 11, pp. 1473-1477, 1998.

[19] O. Voinot, D. Alazard, A. Piquereau, "A robust multiobjective synthesis applied to launcher attitude control", 15th IFAC Symposium on Automatic Control in Aerospace, Bologna, Italy, 2001.

[20] O. Voinot, P. Apkarian, D. Alazard, "Gain-scheduling $H_{\infty}$ control of the launcher in atmospheric flight via linear-parameter varying technique", AIAA Guidance, Navigation and Control Conference, Monterey, California, August 2002. 